\title{
Idiopathic toe walking; to treat or not to treat? that is the question
}

\begin{abstract}
Introduction: Idiopathic toe walking is a diagnosis of exclusion given when a child exhibits persistant toe walking, and all other relevant medical causes of toe walking have been omitted. The purpose of this review is to summarize the knowledge available presently on the etiology and the natural history, and to examine how idiopathic toe walking is evaluated and the effect of current treatments.
\end{abstract}

Method: A systematic search in the PubMed-, Cochrane- and Embase-databases was done, identifying 85 articles, all of which were reviewed.

Results: Currently it seems to be conservative treatment as first choice and surgical intervention as a second choice. The estimated effect of a given treatment, when the parameters used may be subject to measurement uncertainties, can be difficult. Casting and extension of the Achilles tendon are found to be the only treatments to alter the kinematics and kinetics of the ankle joint. The effect is however, relatively short-term and with high recurrence rate. Possibly indicating several etiologies of idiopathic toe walking. Furthermore, the level of evidence for treatment is moderate with predominantly retrospective studies and case-control studies, and few randomized controlled trials.

Conclusion: It is proposed that the possible several etiologies be further examined in a scientific set up to form the basis for finding potentially better etiology based treatment. There is also the need to further clarify the natural history and whether there are long-term complications in such an extent that it continues to justify the current treatments.

Level of evidence: 2 a

Keywords: idiopathic toe walking, 3-D gait analysis, treatment outcomes
Volume 10 Issue 2 - 2018

\author{
Christian Wong,' Merete Speedtberg, ${ }^{2}$ Jesper \\ Bencke, ${ }^{2}$ Emilie Friis' \\ 'Department of Orthopedics, University hospital of Hvidovre, \\ Denmark \\ ${ }^{2}$ Gait laboratory, University hospital of Hvidovre, Denmark
}

Correspondence: Christian Wong, Department of Orthopedics, University hospital of Hvidovre, Kettegaard Allé 30, 2650 Hvidovre, Denmark, Tel +45 3070686I, Fax +45 38626999, Email chwol23@gmail.com

Received: January 23, 2018 | Published: March 08, 2018
Abbreviations: ITW, idiopathic toe walking; AROM, ankle range of motion; AFO, ankle-foot orthosis; FO, foot orthosis; 3DGA, 3-D gait analysis; TAL, chilles tendon

\section{Introduction}

Children usually begin walking at an age of 12-15 months. Initial toe walking is not uncommon until the walk gradually matures into a heel-strike gait pattern. ${ }^{1}$ The stance phase of a normal heel-strike gait pattern entails three phases, ${ }^{1}$ initial contact between heel and the surface, ${ }^{2}$ midfoot contact $a^{3} d^{3}$ the forefoot push off to the swing phase of the gait cycle. ${ }^{1}$ The pattern of toe walking is the involuntary inability or limitation to generate heel-strike during the initial contact phase of the gait cycle., ${ }^{2,3}$ Beyond the age of 2-3 years this lack of heel-strike gait is considered 'pathological toe walking' and could be a peripheral sign of a systemic disease or idiopathic toe walking (ITW) ${ }^{4}$ Children with ITW have the unique characteristic that it is possible for them to briefly change their gait into a heel-strike pattern when asked, thus accomplishing to stand on flat feet - unless they have developed severe contracture of the ankle. ${ }^{5}$

The natural course of ITW has as not yet been fully revealed, and at present there is few documented descriptions. Eastwood et al. ${ }^{6}$ reported 43 out of 49 patients in the observation group still toe walked but had decreased time spent toe walking from $90 \%$ to $60 \% 3$ years after initial evaluation. Stricker and Angulo ${ }^{1}$ observed 48 children with ITW where $25 \%$ of parents reported improvement in gait. Hirsch et al. ${ }^{7}$ supports assumption, that ITW in childhood is a benign condition, which resolves in most instances, with 8 of 11 patients conservatively treated walking normally 7-21 years after diagnosing, and 3 patients exhibited toe walking only when unobtrusively observed.?

The consensus in clinical treating pediatric orthopedics is that most times ITW regresses at the end of growth without severe clinical side effects or complications; heel-to-toe gait is considered spontaneously to commence in early adulthood. ${ }^{11}$ But physician's experience in evaluation and treatment of ITW is usually sparse due to limited encounters for each physician. The etiological background is often only briefly explored before treatment is commenced. ${ }^{1}$ This is reflected in a currently low scientific level of evidence regarding ITW, as the vast majority is case-control studies, retrospective analyses, case studies and only a few randomized controlled trials. ${ }^{[6]}$ Moreover, the published studies are inconsistent in design with varying followup and outcomes, which reduces the comparability between studies.

The purpose of this review is to summarize the present knowledge of ITW with focus on the etiology, natural history, long term consequences and current treatment. Furthermore, we consider how ITW is evaluated by gait analysis and if this method could be used as an evaluation tool for finding new perspectives and proposals to alternate treatments.

\section{Method}

An advanced search was conducted in in the PubMed-database. MeSH words and free text words were divided into six blocks depending on subject: (1) age and gender, (2) gait (and gait 
disturbance), (3) anatomy and physiology and biomechanics, (4) treatment, (5) diagnosis, (6) and treatment evaluation/treatment optimization. Subsequently searches were performed combining the free text words and the MeSH words within each block. Different block search strings were combinations provided search results of 3.925 to 40.607 hits.

It was attempted to decrease the number of hits using filters; search combining all blocks using following filters: review, humans, language (only Danish, Swedish, Norwegian and English); generating 246 hits. All were assessed by title and abstract, but were not found eligible due to the fact that they did not relate to ITW. However, by combining search block 1, 2 and 4 as well as the use of following filters: review, language (only Danish, Swedish, Norwegian and English), child and infant, produced 149 hits. Nine of these were found to be eligible based on title and abstract. Finally, to increase the amount of relevant hits suited for the review, a systematic search in the Pubmed-, Cochrane- and Embase-databases was performed using the free text toe walk*. Earlier, ITW has been referred to as habitual toe walking and as congenital short-tendo-calcaneus. ${ }^{8,9}$ These terms were chosen to ensure inclusion of all relevant publications. The exclusion criteria were publications not written in English, Danish, Norwegian or Swedish, duplicates, casuistic works, animal-studies, studies differentiating ITW and cerebral palsy, and works with no mention of either etiology or treatment. This resulted in 85 articles included for review (Figure 1)

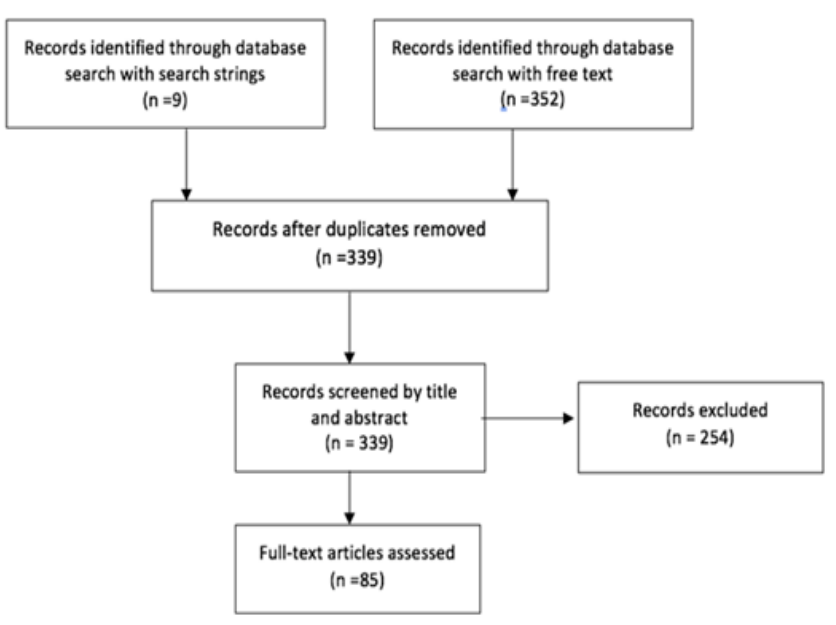

Figure I Flowchart summarizing the selection of relevant articles.

\section{Etiology}

The etiological background for ITW is essentially unknown and a diagnosis of exclusion, therefore other medical causes associated with toe walking must be omitted before the gait pattern is defined as ITW. $2,5,8,10,11$ Suggested factors for ITW has been Hereditary due to positive family history ${ }^{12,13}$ or due to sensory and motor skill deficits or a myopathic disorder. ${ }^{1,3,10,14}$ Despite the varied suggestions as to why seemingly healthy children walk on their toes, the etiology still remains elusive.

\section{Classification}

The multitude of characteristics is reflected in the clinical guide for differential diagnosing toe walking 'the Toe Walking Tool'. With this the physicians are guided through the clinical examination and diagnostic process to confirm the diagnosis of ITW. ${ }^{8}$ The guide
(Figure 2), including birth history, lower limb musculoskeletal, and neurological examination and a developmental screening, ${ }^{10}$ can aid physicians to identify when to refer a toe walking child to further comprehensive assessment, and could be a plausible pathway to find suitable treatment. ${ }^{3}$

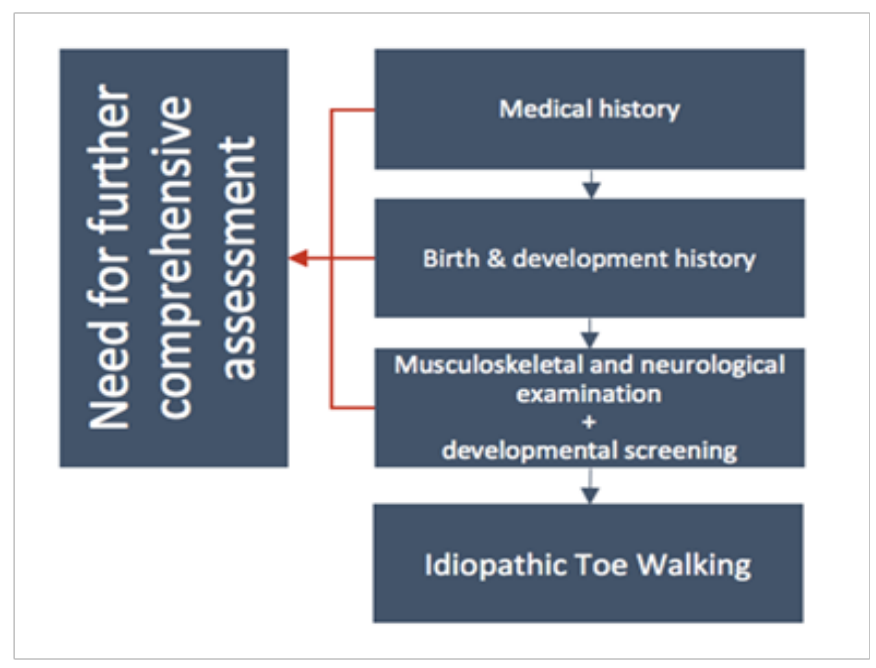

Figure 2 How the 'Toe Walking Tool' guides physicians. Red arrows are answers or findings indicating non-idiopathic cause of toe walking according to the 'ToeWalking Tool' and thus need for further comprehensive assessment.

\section{Clinical evaluation}

The ankle range of motion (AROM) is a parameter commonly used to estimate contractures as primary measure to assess the severity of ITW. However, AROM does not necessarily correlate to the severity of ITW, and cannot reveal a possible improvement in gait pattern. ${ }^{13}$ Furthermore, overall reliability studies show that assessment of passive and active AROM in normal children and young adults has an intra-observer variability with up to 5 to 10 degrees as well as an a high inter-observer variability. ${ }^{15}$ This lack of correlation between the AROM measurements and the gait measures and contracture development diminishes the usefulness of AROM as an evaluation tool for ITW. The 'weight bearing lunge test' is an alternative method of measuring the AROM that has been determined reliable. The patient lunge towards a tapemark on a wall. The distance from the wall (and knee) to the foot correlates with the AROM. ${ }^{8}$

Instead of measuring the AROM due to the poor correlation between AROM and severity of toe walking, functional instrumented evaluation of 3-D gait analysis (3DGA) have been seen as an alternative. A classification of severity of ITW using 3DGA was suggested by Alvarez et al., ${ }^{13}$ where ITW is categorized in three: mild (1), moderate (2) or severe (3) based on three primary criteria determined by ankle kinematics and kinetics: presence of first ankle rocker, presence of early third ankle rocker and predominant first ankle movement. ${ }^{13}$ The classification (Figure 3 ) is meant as a guide for physicians to initiate treatment and monitor the treatment outcome. Even though 3DGA provides more detailed data on the gait, the children can adopt normal heel-strike gait when being observed, ${ }^{4,5,15,16}$ which makes assessment of the degree of toe walking difficult. This would obscure their 'normal' toe walk, and therefore hide the true efficacy of a given treatment or the true severity of their condition. These challenges have to be overcome in relation to assessment and management of ITW. 


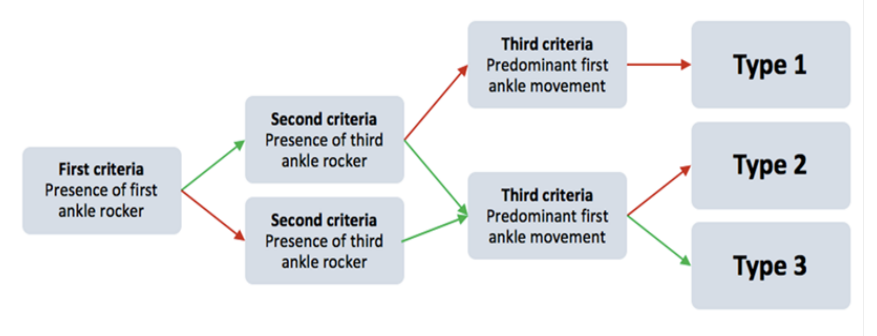

Figure 3 How to classify severity by the three primary criteria. Green arrows illustrating 'Yes' and red arrows illustrating 'No'.

\section{Natural history and complications, treatments and outcomes}

There seem no evidence that children with ITW will acquire poor functional outcomes if continued to toe walk into adolescence. ${ }^{[1]}$ However, ankle contracture is described as a negative effect of longterm toe walking. ${ }^{4,16,17}$ According to van Bemmel et al. ${ }^{16} 50$ to $70 \%$ of children with ITW had measurable ankle contracture, and according to Williams et al. ${ }^{8}$ ITW is associated with a mild to greatly reduced available AROM compared to normal children. ${ }^{8}$ This is opposed to Hirsch et al. ${ }^{14}$ where no long-term fixed ankle contracture during or after conservative treatment was detected at (7-21 years) follow-up. Solan et al. ${ }^{11}$ states that whether or not persistent toe walking results in true shortening of the calf over time, the development of contractures in children with ITW is of importance since equinus contractures in adulthood potentially could lead to foot and ankle pathologies. ${ }^{16,18}$

Furthermore, it is suggested that ITW is associated with lumbar spine hyperlordosis, undifferentiated leg pain, limping, fatigue, and the possibility of developing external tibial torsion to compensate for the lack of foot flat contact, as well as forefoot splay and disproportionately wide forefoot and narrow heel..$^{2,4,18}$ Finally, Herrin et al. ${ }^{4}$ report short-term complications of ITW as an immediate negative impact on the spatiotemporal gait parameters, that subsequent would lead to increased risk of falling. Shorter stride length and a lower cadence would result in the child using greater effort to walk than their peers with normal gait. ${ }^{4}$ The treatments available today include observation, non-surgical intervention by physical therapy, orthoses, serial casting, injections of Botulinum toxin A and surgical release by Achilles tenotomy. ${ }^{1}$ A common denominator for the current treatment methods is that they are aimed at improving AROM. The rationale is that when achieving AROM within normal range, it will cause the children with ITW to adopt a heel-strike gait, and subsequent prevent the presumed complications of the condition. A treatment protocol is suggested according to the Alvarez classification system; Type 1 and 2 are recommended observation only, with a clinically reassessment for type 1 and with gait analysis for type 2, if their toe-walking issues continuous complaints. For the type 3 treatment of either serial casting, Botulinum Toxin A, orthoses, physical therapy was recommended for the younger and/or surgery for the older children. ${ }^{13}$ In the following the different treatments for ITW will be described:

\section{Observation}

Eastwood et al (2000) compared watchful waiting with cast and surgery treatment. ${ }^{6}$ The outcome of the observation and cast had no significant difference; $88 \%$ of the observation group were still toewalking at a mean follow-up at 3,2 years, and $78 \%$ of the cast group toe-walked at a mean follow-up at 3,7 years.

\section{Physical therapy}

Physical therapy for treating ITW is exercises that exert stretch of the gastrocnemius and soleus complex. The existing studies are retrospective and often comparing physical therapy in combination with other conservative treatments. ${ }^{1,7,13}$ Physiotherapy shows an improvement in AROM immediately after, but the effect seems to be relatively short-term and unable to induce permanent change to the children's gait pattern. ${ }^{7,13}$ The effects of adding physical therapy to the period of casting does not seem to have any lasting effect on toe walking ${ }^{1,6}$ Hirsch et al. ${ }^{7}$ found no difference in 10 out of 11 patients. ${ }^{7}$

\section{Orthoses}

Herrin \& Geil ${ }^{4}$ examined a 6 weeks use of two mechanical different orthoses on 18 patients for immediate 'carry-over effects'; day time use of an ankle-foot orthosis (AFO) or a foot orthosis (FO). The AFO blocked ankle plantarflexion and resisted toe flexion inducing a mechanical inhibition of toe-walking and the FO was a supramalleolar orthosis combined with a rigid carbon fiber footplate. This combination was unrestrictive in a mechanical sense and sought to impede toe walking by treating a sensory-perceptual equinus. ${ }^{4} \mathrm{Heel}$ strike during gait was obtained fully when wearing AFO's opposed to $87 \%$ for the FO-foot plate. However, the effect of these treatments did not improve toe walking significantly compared to the shoes only condition after treatment. There was significant improvement after treatment in reducing early heel rise in the FO-foot plate group, which was not found in the AFO group; this could indicate that focusing treatment of the sensory-perceptual component instead of the strict mechanical component would be more effective. ${ }^{4}$

\section{Botulinum toxin A}

Botulinum toxin A injections have a temporary paralytic effect of the calf muscles, thus facilitating heel strike by preventing contraction of the calf muscles. The treatment was combined with physical therapy both with and without simultaneously serial casting. Engström et al. ${ }^{19}$ performed a randomized controlled trial which showed no improvement when supplementing injection of Botulinum toxin A to cast treatment, and recommended that injection treatment was omitted. ${ }^{19}$

\section{Casting}

Serial casting below the knee is used to alleviate ankle contracture by applying plaster casts in various and increasing degree of dorsiflexion for a period of 3-10 weeks. ${ }^{6,715,16,19,20}$ Engström et al. ${ }^{19}$ performed a small randomized controlled study in which 38 out of 47 children were still toe walking 3 and 12 months after casting.

Significant improvements were seen in gait pattern and these were seen especially at the 3 month evaluation and were accredited the casting treatment. ${ }^{19}$ This is supported by Fox et al. ${ }^{20}$ where parents reported $66 \%$ normal walking or satisfactory improvement after casting for children. Stott et al (2004) ${ }^{[18]}$ found changes in sagittal plane ankle kinematics after casting treatment for ITW in 12 out of 13 patients skeletal mature. ${ }^{[18]}$ Even though casting seems to induce lasting effects on the ankle kinematics, other authors consider these temporary and ineffective. Hirsch et al. ${ }^{7}$ found that 10 out of 11 subjects had recurring toe walking patterns at initial evaluation after casting, and Eastwood et al. ${ }^{6}$ found no long term change between the observation- and cast-treated group as described earlier. 
Reported complications from casting were few and relative mild, including pain during casting and pressure sores. ${ }^{7,20}$

\section{Surgical intervention}

Surgical intervention has generally been used in cases with fixed ankle contractures with limited dorsiflexion and/or if conservative treatment has been unsuccessful. ${ }^{1,21}$ The surgical procedure improves the heel-toe gait with lengthening of the triceps surae muscletendon complex, which enables an increase in dorsiflexion in stance- and swing-phase. ${ }^{1}$ This procedure can be performed as open or percutaneously with several techniques as proximal intrafascial lengthening or as a distal tenotomy in the Achilles tendon (TAL). ${ }^{1,11}$ These procedures change kinematics and kinetics at ankle and knee level in the sagittal plane with improvement in dorsiflexion in stance and swing, increase of peak knee flexion in swing, longer stride length and decrease in cadence. ${ }^{21}$ McMulkin et al..$^{22}$ reported a persisting effect both at 1 year follow-up and after more than 5 years. ${ }^{17,21,22}$ Although inducing changes in gait patterns, surgery does not normalize the gait completely, since only $37 \%$ had normal gait after surgery at time of follow-up (mean follow-up was 7,9 years). ${ }^{6}$ A review by van Bemmel et al. ${ }^{16}$ compared 10 studies with surgery or casting; their conclusion was that no clear advantage of neither cast nor surgery as treatment for ITW. However, they found that surgery did improve dorsiflexion for equinus contracture.

Complications from surgical procedures are more severe than for casting with overlengthening and calcaneal gait, mild pain during long duration activities, Achilles tendinitis, superficial wound infection, and one case of ankle fracture during percutaneous gastrocnemius lengthening. $1,16,22$

\section{Discussion}

Based on this review the general approach to treating the condition ITW is the non-invasive method as casting or watchful waiting as a first choice. If the results after non-invasive management are unsatisfactory, if the child is older or presenting ankle contracture, surgery is next treatment of choice. ${ }^{16}$ The most successful treatments of current methods seem to be casting and surgery. Casting often offers at least short-term effect with few and relative mild complications. On the other hand surgery improves kinematics and kinetics on ankle and knee level more than casting with longer effect. ${ }^{22}$ Moreover, surgery is a superior treatment for severe ankle contracture, but the complications are also more severe than those of casting. ${ }^{1}$ Both casting and surgery improve the walking pattern in children with ITW when analyzed with gait analysis, but heel strike gait is not achieved as for their peers, and parental-perceived gait shows temporary improvement, but no long term satisfactory result. We speculate if this might be due to the fact that current treatment methods plainly do not work. However, some children with ITW benefit from treatment as described earlier. The apparent lack of good long term treatment results indicate, that the apparent heterogeneous group of children might need a 'heterogeneous' treatment as well with either specific deficit oriented treatments or with a multimodal approach. Research into sensory processing, vibration threshold and motor skill attainment of children with ITW suggests that at least some of the children with ITW do not have an isolated pathology in the ankle joint or adjacent anatomical regions, but have multifocal etiologies or yet again an isolated cerebral causality., ${ }^{3,10,23}$ This could explain the ambiguous treatment results. We would suggest, that instead of looking at ITW as a diagnosis of exclusion, one should look into the plausible etiologies to ITW and if found, subsequent propose an etiological based treatment. If in fact these children require different methods of treatment, the physician firstly need to distinguish between the various etiological subgroups (strictly motor control, sensory processing or a mixed sensorimotor problem), and this leaves us with a challenge of how to differentiate the patients in adequate subgroups. The perspective of the above considerations is to find novel treatments. One possibility may be through research into sensory processing of children with ITW, since children with ITW display more sensory processing difficulties than typically developing children, as mentioned previously. ${ }^{23,24}$

There are certain obstacles in research and treatment of ITW. One consideration is to document the outcome uniformly and systematically of current and future treatments. There are uncertainties in regards to which parameters to record and measuring bias of for example AROM and 3DGA parameters. Currently, a possible correlation between ITW and an effective treatment might not be detected. Another consideration is low number of potential ITW children to recruit for a randomized design study with several treatments. At present the bulk of research is retrospective studies and case-control studies with only a few published randomized controlled trials. The level of evidence for treatment must be considered moderate with an obvious need of undertaking studies to enlighten the etiological background for ITW and the efficacy of current treatments. Moreover, studies with long term follow up should be undertaken to see, if ITW indeed have long term health consequences to ensure if treatment is needed at all. The issue to treat or not to treat is still a pressing matter as long as the natural history is insufficiently mapped and the long-term complications are unknown.

\section{Conclusion}

ITW is a challenge with many aspects to consider and overcome in regard to treating these children. Presently casting and surgery seems to be the best methods of managing the condition, though walking is not normalized but improved. This could suggest the existence of multiple unknown etiologies instead of only one, and some may possibly be secondary to others. Improvement in clinical subclassification of these children seem to be a viable pathway as a first step to find a more effective to ensure etiology based treatment(-s). Other challenges may be to map the natural history including longterm complications to see if treatment is needed at all.

\section{Compliance with ethical Standards}

This article does not contain any studies with human participants or animals performed by any of the authors.

There are no conflicts of interest and funding was not received. This was a literature review and did not involve human participants and/or animals, and informed consent was not needed.

\section{Acknowledgements}

None.

\section{Conflict of interest}

Authors have declared no conflicts in publishing the manuscript.

\section{References}

1. Oetgen ME, Peden S. Idiopathic toe walking. J Am Acad Orthop Surg. 2012;20(5):292-300. 
2. van Kuijk AAA, Kosters R, Vugts M, et al. Treatment for idiopathic toe walking: A systematic review of the literature. $J$ Rehabil Med. 2014;46(10):945-957.

3. Williams CM, Tinley $\mathrm{P}$, Curtin M, et al. Vibration perception thresholds in children with idiopathic toe walking gait. $J$ Child neurol. 2011;27(8):1017-1021

4. Herrin K, Geil M. A comparison of orthoses in the treatment of idiopathic toe walking: A randomized controlled trial. Prosthet orthot int. 2016;40(2):262-269.

5. Ruzbarsky JJ, Scher D, Dodwell E. Toe walking: causes, epidemiology, assessment, and treatment. Curr Opin Pediatr. 2016;28:40-46

6. Eastwood DM, Menelaus MB, Dickens DRV, et al. Idiopathic toewalking: Does treatment alter the natural history? J pediatr orthop $B$. 2000;9:47-49.

7. Hirsch G, Wagner B. The natural history of idiopathic toe-walking: a long-term follow-up of fourteen conservatively treated children. Acta Paediatr. 2004;93:196-199.

8. Williams, CM et al. Idiopathic toe walking: Have we progressed in our knowledge of the causality and treatment of this gait type? J Am Podiatr Med Assoc. 2014;104:253-262.

9. Sala DA, Shulman LH, Kennedy RF, et al. Idiopathic toe-walking: A review. Dev Med Child Neurol. 1999;41:846-848.

10. Williams, CM Tinley $\mathrm{P}$, Curtin $\mathrm{M}$, et al. Is idiopathic toe walking really idiopathic? The Motor skills and sensory processing abilities associated with idiopathic toe walking gait. J Child Neurol. 2014;29:71-78.

11. Solan MC, Kohl-Gatzoulis J, Stephens MM. Idiopathic toe walking and contractures of the triceps surae. Foot Ankle Clin. 2010;15:297-307.

12. Pomarino D, Llamas JR, Pomarino A. Idiopathic toe walking: Family predisposition and gender distribution. Foot Ankle spec. 2016;9:417-422.

13. Alvarez C, De Vera M, Beauchamp R, et al. Classification of idiopathic toe walking based on gait analysis: Development and application of the ITW severity classification. Gait Posture. 2007;26:428-435.
14. Williams CM, Pacey V, Bakker PB, et al. Interventions for idiopathic toe walking (protocol). Cochrane Database of Systematic Reviews. 2016;10:CD012363.

15. Stott NS, Walt SE, Lobb GE, et al. Treatment for idiopathic toe-walking results at skeletal maturity. J Pediatr Orthop. 2004;24:63-69.

16. Bemmel AF, Graaf VA, van den Bekerom MPJ, et al. Outcome after conservative and operative treatment of children with idiopathic toe walking: a systematic review of the literature. Musculoskelet Surg. 2014;98:87-93.

17. Hemo Y, Macdessi SJ, Pierce RA, et al. Outcome of patients after achilles tendon lengthening for treatment of idiopathic toe walking. $J$ Pediatr Orthop. 2006;26:336-340.

18. Dietz F, Khunsree S. Idiopathic toe walking: To treat or not to treat, that is the question. Iowa Orthop J. 2012;32:184-188.

19. Engström P, Tedroff K, Orefelt C, et al. Botulinum Toxin A does not improve the results of cast treatment for idiopathic toe-walking. $J$ Bone Joint Surg Am. 2013;95:400-407.

20. Fox A, Deakin S, Pettigrew G, et al. Serial casting in the treatment of idiopathic toe-walkers and review of the literature. Acta Orthop Belg. 2006;72:722-730.

21. McMulkin ML, Baird GO, Caskey PM, et al. Comprehensive outcomes of surgically treated idiopathic toe walkers. J Pediatr Orthop. 2006;26:606611.

22. McMulkin ML, Gordon AB, Tompkins BJ, et al. Long term gait outcomes of surgically treated idiopathic toe walkers. Gait Posture. 2016;44:216 220.

23. Williams CM, Michalitsis J, Murphy AT, et al. Whole-body vibration results in short-term improvement in the gait of children with idiopathic toe walking. J Child Neurol. 2016;31:1143-1149.

24. Baber S, Michalitsis J, Fahey M, et al. A comparison of the birth characteristics of idiopathic toe walking and toe walking gait due to medical reasons. J Pediatr. 2016;171:290-293. 